\title{
Research Article \\ Experimental Active Vibration Control in Truss Structures Considering Uncertainties in System Parameters
}

\author{
Douglas Domingues Bueno, Clayton Rodrigo Marqui, Rodrigo Borges Santos, \\ Camilo Mesquita Neto, and Vicente Lopes Jr.
}

Grupo de Materiais e Sistemas Inteligentes (GMSINT), Departamento de Engenharia Mecânica (DEM), Faculdade de Engenharia de Ilha Solteira (FEIS), Universidade Estadual Paulista (UNESP), Avenida Brasil Centro 56, 15385-000 Ilha Solteira, SP, Brazil

Correspondence should be addressed to Vicente Lopes Jr., vicente@dem.feis.unesp.br

Received 16 October 2007; Revised 11 June 2008; Accepted 9 August 2008

Recommended by Paulo Gonçalves

This paper deals with the study of algorithms for robust active vibration control in flexible structures considering uncertainties in system parameters. It became an area of enormous interest, mainly due to the countless demands of optimal performance in mechanical systems as aircraft, aerospace, and automotive structures. An important and difficult problem for designing active vibration control is to get a representative dynamic model. Generally, this model can be obtained using finite element method (FEM) or an identification method using experimental data. Actuators and sensors may affect the dynamics properties of the structure, for instance, electromechanical coupling of piezoelectric material must be considered in FEM formulation for flexible and lightly damping structure. The nonlinearities and uncertainties involved in these structures make it a difficult task, mainly for complex structures as spatial truss structures. On the other hand, by using an identification method, it is possible to obtain the dynamic model represented through a state space realization considering this coupling. This paper proposes an experimental methodology for vibration control in a 3D truss structure using PZT wafer stacks and a robust control algorithm solved by linear matrix inequalities.

Copyright (C) 2008 Douglas Domingues Bueno et al. This is an open access article distributed under the Creative Commons Attribution License, which permits unrestricted use, distribution, and reproduction in any medium, provided the original work is properly cited.

\section{Introduction}

Lightweight space structures are the future of space vehicles and satellite technology. Possessing ideal space launching characteristics, such as minimal storage volume and minimal mass, these lightweight structures will propel the space industry into the next generation. Space satellites must be expertly controlled from a vibration standpoint because signal transmission to and from the earth mandates tight tolerances. Vibration control is critical to mission success as well as satellite longevity [1]. 
Large and light space structures are basically flexible due to their low stiffness and damping. These characteristics may cause problems since flexible structures present many vibration modes within or beyond the bandwidth of the controller. When only a few modes are dealt in the controller, spillover may occur because uncontrolled higher modes or unmodeled modes may become excited. The effects of spillover can occur where structure, sensors, or actuators are poorly modeled, and the numbers of sensors and actuators are low.

In order to achieve better dynamic properties, great attention has been paid to the control of structural vibration using intelligent structures. So, the application of active vibration control in flexible structures has been increasingly used as a solution for space structures to achieve the degree of vibration suppression required for precision pointing accuracy and to guarantee the stability.

In truss structures, active members are integrated because of their multiple functions. They serve as structural elements, and as load device. As actuators, the active member exerts internal forces, and as sensor elements, it allows measurement of the elastic strains. The piezoelectric stack actuators are remarkable because they are light weight, high force, and low power consumption [2].

Several researchers have proposed the use of piezoelectric material for active vibration control. In truss structures, the control force can be accomplished by piezoelectric active members, known as "PZT wafer stacks," that are mechanically linked in series producing an axial force in the bar that are positioned.

State space realization is used in modern control formulation to obtain the dynamic model, but in many cases this model has significant uncertainties in relation to the real system. These uncertainties can be caused, for instance, by parameter variation during the operation, or by dynamic uncertainties (nonlinearities, higher modes, noises, etc.). So, for an efficient experimental control design, it is important to qualify and quantify the uncertainties.

In this context, this paper proposes a methodology for robust control design considering uncertainties in the dynamic model, represented by state space realization. It is designed as an active controller to attenuate vibrations in a truss structures. The active members are composed by PZT wafer stacks actuators, and the control design is based on linear quadratic regulator solved through linear matrix inequalities (LMIs). LMI presents advantages when compared to conventional techniques, and it has contributed to overcome many difficulties in control design [3]. In the last decade, LMI has been used to solve many problems that until then were unfeasible through other methodologies, mainly due to the emergence of powerful algorithms to solve convex optimization problem, for instance, the interior point method (see Boyd et al. [4] and Gahinet et al. [5]). Sarracini and Serpa [6] apply Hinfinite control approach solved through LMI for model reduction. Silva et al. [7] present a consistent formulation for control design based on LMI approach. Chen and Zhu [8] present a formulation based on $H_{2}, H_{\infty}$, and mixed $H_{2} / H_{\infty}$ control strategies for a flexible rotor system under seismic excitation by means of linear matrix inequality (LMI) to attenuate the transient vibration of the flexible rotor system under a nonstationary seismic excitation and to improve robust performance of the flexible rotor system.

In the present work, the numerical method of subspace and the Kalman estimator were used to identify the dynamic model with experimental data and to estimate the state vector, respectively. Experimental results, obtained through dSPACE control board and the Simulink/Matlab, are shown in order to validate the proposed approach. 


\section{Dynamic modeling of robust control}

Modern linear control formulation is based on the state space realization. Using this formulation, the design of a vibration control for multi-input multioutput (MIMO) system is similar of simple-input simple-output (SISO) system approach. This realization is appropriate for experimental applications because there are many numerical methods to solve it in real time, as for instance, the numerical method of Runge-Kutta. A linear differential inclusion (LDI) system, in modal state space form, considering the matrices with appropriate dimensions and assumed to be known, is given by

$$
\begin{gathered}
\dot{\mathbf{x}}(t)=\mathbf{A}(t) \mathbf{x}(t)+\mathbf{B}_{w}(t) \mathbf{w}(t)+\mathbf{B}_{u}(t) \mathbf{u}(t)+\mathbf{E}_{\text {in }} \mathbf{v}_{\text {in }}(t), \\
\mathbf{y}(t)=\mathbf{C}(t) \mathbf{x}(t)+\mathbf{D} \mathbf{u}(t)+\mathbf{E}_{\text {out }} \mathbf{v}_{\text {out }}(t), \\
{\left[\begin{array}{cc}
\mathbf{A}(t) & \mathbf{B}_{w}(t) \\
\mathbf{B}_{u}(t) & \mathbf{C}(t)
\end{array}\right] \in \mathbf{\Omega},}
\end{gathered}
$$

where $\boldsymbol{\Omega}$ is a polytope that is described by a list of vertexes in a convex space, $\mathbf{A}(t)$ is the dynamic matrix, $\mathbf{B}_{w}(t)$ is the matrix of disturbance, $\mathbf{B}_{u}(t)$ is the matrix of control input, $\mathbf{C}(t)$ is the output matrix, $\mathbf{w}(t)$ is the vector of disturbance input, $\mathbf{u}(t)$ is the vector of control input, $\mathbf{y}(t)$ is the output vector, and $\mathbf{v}_{\text {in }}(t)$ and $\mathbf{v}_{\text {out }}(t)$ are stationary zero-mean Gaussian white process and measurement noises vectors with unit intensity, respectively. In this paper, some variables, as the matrices in (2.1), are represented as time function to emphasize the uncertainties in the system parameters. The vectors $\mathbf{E}_{\mathrm{in}}$ and $\mathbf{E}_{\text {out }}$ are the process and measurement noise vectors, respectively.

The state vector $\mathbf{x}(t)$ of the modal coordinates system consists of $n$ independent components, $\mathbf{x}_{i}(t)$, that represent a state of each mode, where $n$ is the number of modes. The $\mathbf{x}_{i}(t)$ (ith state component), related to (2.3), is defined as [9]

$$
\mathbf{x}_{i}(t)=\left\{\begin{array}{c}
\mathbf{q}_{m i}(t) \\
\mathbf{q}_{m o i}(t)
\end{array}\right\}, \quad \text { where } \mathbf{q}_{m o i}(t)=\zeta_{i}(t) \mathbf{q}_{m i}(t)+\frac{\dot{\mathbf{q}}_{m i}(t)}{\omega_{i}(t)}
$$

where $\mathbf{q}_{m i}$ and $\mathbf{q}_{m o i}$ are named modal displacement and velocity for $i$ th vibration mode, respectively. Using modal coordinates, these parameters have no physical interpretation. Also, $\zeta_{i}$ and $\omega_{i}$ are damping factor and natural frequency of the $i$ th mode. These parameters are represented as time function to emphasize the uncertainties.

The modal state space realization is characterized by the block-diagonal dynamic matrix and the related input and output matrices [9]:

$$
\begin{aligned}
& \mathbf{A}(t)=\operatorname{diag}\left(\mathbf{A}_{m i}(t)\right), \quad \mathbf{B}_{w}(t)=\left[\begin{array}{c}
\mathbf{B}_{w m 1}(t) \\
\mathbf{B}_{w m 2}(t) \\
\vdots \\
\mathbf{B}_{w m n}(t)
\end{array}\right], \quad \mathbf{B}_{u}(t)=\left[\begin{array}{c}
\mathbf{B}_{u m 1}(t) \\
\mathbf{B}_{u m 2}(t) \\
\vdots \\
\mathbf{B}_{u m n}(t)
\end{array}\right], \\
& \mathbf{C}(t)=\left[\begin{array}{llll}
\mathbf{C}_{m 1}(t) & \mathbf{C}_{m 2}(t) & \cdots & \mathbf{C}_{m n}(t)
\end{array}\right],
\end{aligned}
$$


where $i=1,2, \ldots, n ; \mathbf{A}_{m i}, \mathbf{B}_{w m i}, \mathbf{B}_{u m i}$, and $\mathbf{C}_{m i}$ are $2 \times 2,2 \times k, 2 \times s$, and $r \times 2$ blocks, respectively; $k$ is the number of disturbances; $s$ is the number of control inputs; and $r$ is the number of outputs. These blocks can be obtained in several different forms and also it is possible to convert it in another realization through a linear transformation. One possible form to block $\mathbf{A}_{m i}(t)$ is

$$
\mathbf{A}_{m i}(t)=\left[\begin{array}{cc}
-\zeta_{i}(t) \omega_{i}(t) & \omega_{i}(t) \\
-\omega_{i}(t)\left(\zeta_{i}^{2}(t)-1\right) & -\zeta_{i}(t) \omega_{i}(t)
\end{array}\right]
$$

The dynamic model of the truss structure was initially identified using experimental data through subspace identification method. $n_{I}>1$ identification tests were considered to characterize the uncertainties in the system parameters. The members that include PZT stack actuator are nominated by active members. It is considered a variation in the properties of these members caused by the insertion of these actuators. These uncertainties are described by a polytopic linear differential inclusion (PLDI):

$$
\begin{aligned}
\boldsymbol{\Omega}= & {\left[\begin{array}{ll}
\mathbf{A}_{c}(t) & \mathbf{B}_{w c}(t) \\
\mathbf{B}_{u c}(t) & \mathbf{C}_{c}(t)
\end{array}\right], } \\
& \operatorname{Co}\left\{S_{1}, \ldots, S_{v}\right\}, \\
\text { where } S_{i}= & {\left[\begin{array}{cc}
\mathbf{A}_{c i} & \mathbf{B}_{w c i} \\
\mathbf{B}_{u c i} & \mathbf{C}_{c i}
\end{array}\right], \quad i=1, \ldots, v, }
\end{aligned}
$$

where the subscript $c$ is relative to controlled modes; $\boldsymbol{\Omega}$ is a polytope described by a list of vertexes in a convex space Co [4], and $v$ is the number of vertexes of the polytopic system. Usually, in practical situations, it is very difficult to define the polytopic vertexes, but these vertexes are not variant in time. So, it is possible to project the vibration control using an invariant model.

A reduced-order model is obtained by truncating the states. Let $\mathbf{x}(t)$ and the state $(\mathbf{A}(t)$, $\left.\mathbf{B}_{w}(t), \mathbf{B}_{u}(t), \mathbf{C}(t)\right)$ be partitioned considering the canonical modal decomposition. From the Jordan canonical form, the following can be obtained :

$$
\begin{aligned}
\left\{\begin{array}{c}
\dot{\mathbf{x}}_{c}(t) \\
\dot{\mathbf{x}}_{r}(t)
\end{array}\right\} & =\left[\begin{array}{cc}
\mathbf{A}_{c}(t) & 0 \\
0 & \mathbf{A}_{r}(t)
\end{array}\right]\left\{\begin{array}{l}
\mathbf{x}_{c}(t) \\
\mathbf{x}_{r}(t)
\end{array}\right\}+\left[\begin{array}{c}
\mathbf{B}_{w c}(t) \\
\mathbf{B}_{w r}(t)
\end{array}\right] \mathbf{w}(t)+\left[\begin{array}{c}
\mathbf{B}_{u c}(t) \\
\mathbf{B}_{u r}(t)
\end{array}\right] \mathbf{u}(t), \\
\mathbf{y}(t) & =\left[\begin{array}{ll}
\mathbf{C}_{c}(t) & \mathbf{C}_{r}(t)
\end{array}\right]\left\{\begin{array}{c}
\mathbf{x}_{c}(t) \\
\mathbf{x}_{r}(t)
\end{array}\right\},
\end{aligned}
$$

where $\mathbf{A}_{c}(t)$ is given by (2.4) and the subscript $r$ is relative to the residual modes. Generally, in practical applications, $\mathbf{E}_{\mathrm{in}}$ and $\mathbf{E}_{\text {out }}$ are not identified, but there are, always, some process and measurement noises.

\section{Control methodology}

In this section, a robustness analysis is conduced for understanding the LQR-LMI controller performance. Controller design can be done through rigorous mathematical optimization 
techniques. One of these, which was originated in the sixties [10], is called modern optimal control theory that is a time-domain technique.

Control systems robustness is defined as the ability for maintaining satisfactory stability and performance features in the presence of parameters variations and uncertainties [11]. Traditional LQR solved by Riccati's equation can be obtained in text books [12]. In the following, one presents the procedure for the LQR-LMI approach.

Firstly, mathematical definitions of some terms are given, and LQR control problem is defined. Then, the LQR problem is represented as an equivalent eigenvalue problem (EVP) in terms of LMI using the $\mathrm{H}_{2}$ representation of the LQR problem.

\subsection{Basic definitions of LMIs and EVPs}

A linear matrix inequality has the form

$$
\mathbf{F}(\mathbf{z})=\mathbf{F}_{0}+\sum_{i=1}^{m} \mathbf{z}_{i} \mathbf{F}_{i}>\mathbf{0}
$$

where $\mathbf{z}$ is a real vector and $\mathbf{F}_{0}$ and $\mathbf{F}_{i}$ are real symmetric matrices. Inequality (3.1) is shorthand for saying that $\mathbf{F}(\mathbf{z})$ is positive definite. A vector $\mathbf{z}$ that satisfies inequality (3.1) is known as a feasible solution of the LMI.

Inequality (3.1) is a convex constraint on $\mathbf{z}$. This property is important because powerful numerical techniques are available for the solution of problems involving convex LMIs [4]. On the other hand, no efficient algorithm is available for the solution of nonconvex problems. Hence, nonconvex inequalities which may arise from a control problem should be converted to convex LMIs to be solvable numerically. One useful example for such manipulations is the LMI representation of the following nonconvex inequalities:

$$
\mathbf{Q}(\mathbf{z})-\mathbf{S}(\mathbf{z}) \mathbf{R}(\mathbf{z})^{-1} \mathbf{S}^{T}(\mathbf{z})>\mathbf{0}, \quad \mathbf{R}(\mathbf{z})>0,
$$

where $\mathbf{Q}, \mathbf{R}$, and $\mathbf{S}$ are affine functions of $\mathbf{z}$, and $\mathbf{Q}$ and $\mathbf{R}$ are symmetric matrix. Inequality (3.2) is equivalent to

$$
\left[\begin{array}{cc}
\mathbf{Q}(\mathbf{z}) & \mathbf{S}(\mathbf{z}) \\
\mathbf{S}^{T}(\mathbf{z}) & \mathbf{R}(\mathbf{z})
\end{array}\right]>\mathbf{0}
$$

This transformation can be achieved easily premultiplying inequality (3.3) by

$$
\left[\begin{array}{cc}
\mathrm{I} & -\mathrm{SR}^{-1} \\
0 & \mathrm{I}
\end{array}\right]>0
$$

and postmultiplying it by its transpose. 
One of the concepts related with LMIs and control problems is the eigenvalue problem. An eigenvalue problem may have several representations, one of which is given by

$$
\begin{array}{ll}
\min & \mathbf{c}^{T} \mathbf{z} \\
\text { subject to } & \mathbf{A}(\mathbf{z})>\mathbf{0},
\end{array}
$$

where $\mathbf{A}(\mathbf{z})$ is a symmetric and affine function of $\mathbf{z}$. An LQR problem may be transformed into this form of the EVP to be represented in terms of LMIs.

\subsection{LQR control problem}

There are various representations of LQR problem in the literature. Here, definitions are given in a way that aids the derivation of the specific problem defined above; hence they may not be the most general forms of $L Q R$ problem. The LQR problem is to find the control gain $\mathbf{K}$ that satisfies the optimization

$$
\begin{array}{ll}
\min _{\mathbf{K}} & \mathrm{E}\left[\mathbf{y}^{T} \tilde{\mathbf{Q}} \mathbf{y}+\mathbf{u}^{T} \tilde{\mathbf{R}} \mathbf{u}+\mathbf{y}^{T} \tilde{\mathbf{N}} \mathbf{u}+\mathbf{u}^{T} \tilde{\mathbf{N}}^{T} \mathbf{y}\right], \\
\text { subject to } & (2.1) \text { and } \mathbf{u}(t)=-\alpha \mathbf{K} \mathbf{x}(t),
\end{array}
$$

where $\widetilde{\mathbf{Q}} \geq 0$ and $\widetilde{\mathbf{R}}>0$ are symmetric weighting matrices, $\widetilde{\mathbf{N}}$ is the weighting matrix between input and output vectors, and $\alpha$ is a scalar amplifier. Substituting the output equation in (2.1) into the optimization problem (3.6), and assuming $\mathbf{E}_{\text {in }}$ and $\mathbf{E}_{\text {out }}$ as zero vectors; one obtains another form of the LQR problem

$$
\begin{array}{ll}
\min _{\mathbf{K}} & \mathrm{E}\left[\mathbf{x}^{T} \mathbf{Q} \mathbf{x}+\mathbf{u}^{T} \mathbf{R} \mathbf{u}+\mathbf{x}^{T} \mathbf{N u}+\mathbf{u}^{T} \mathbf{N}^{T} \mathbf{x}\right], \\
\text { subject to } & \dot{\mathbf{x}}(t)=\mathbf{A}(t) \mathbf{x}(t)+\mathbf{B}_{w}(t) \mathbf{w}(t)+\mathbf{B}_{u}(t) \mathbf{u}(t), \quad \mathbf{u}(t)=-\alpha \mathbf{K} \mathbf{x}(t),
\end{array}
$$

where

$$
\mathbf{Q}=\mathbf{C}^{T} \tilde{\mathbf{Q}} \mathbf{C}, \quad \mathbf{N}=\mathbf{C}^{T} \tilde{\mathbf{Q}} \mathbf{D}+\mathbf{C}^{T} \tilde{\mathbf{N}}, \quad \mathbf{R}=\widetilde{\mathbf{R}}+\mathbf{D}^{T} \tilde{\mathbf{Q}} \mathbf{D}+\mathbf{D}^{T} \tilde{\mathbf{N}}+\tilde{\mathbf{N}} \mathbf{D} .
$$

\subsection{EVP representation of the LQR problem}

Lyapunov's stability criteria can be used to state that a system given by (2.1) with control force $\mathbf{u}(t)=-\alpha \mathbf{K x}(t)=-\mathbf{G} \mathbf{x}(t)$, where $\mathbf{G}=\alpha \mathbf{K}$, is stable if there exists a matrix $\mathbf{S}=\mathbf{S}^{T}>\mathbf{0}$ that satisfies

$$
\left(\mathbf{A}-\mathbf{B}_{u} \mathbf{G}\right) \mathbf{S}+\mathbf{S}\left(\mathbf{A}-\mathbf{B}_{u} \mathbf{G}\right)^{T}+\mathbf{B}_{w} \mathbf{B}_{w}^{T} \leq 0
$$




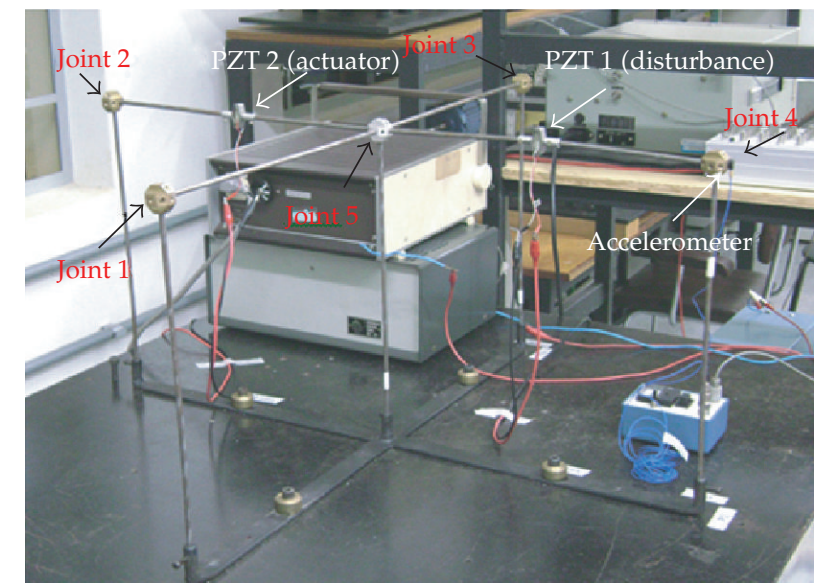

(a)

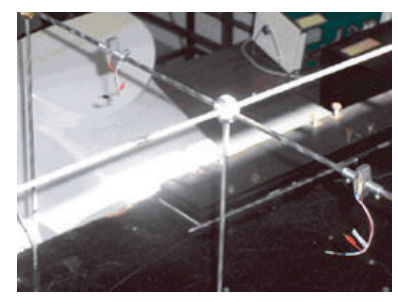

(b)

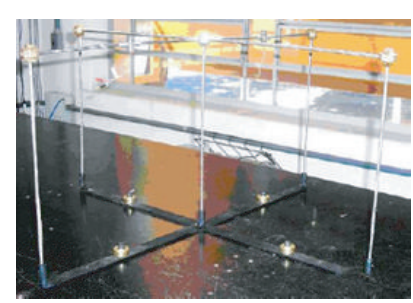

(c)

Figure 1: 3D truss structures and PZT wafer stack actuators.

where $\mathbf{S}$ that satisfies inequality (3.9) is the optimal state covariance matrix $\mathbf{P}$. So, combining (3.7) and (3.9) and optimizing $\mathbf{S}$, the $H_{2}$ optimization problem may be stated as [13]

$$
\begin{array}{ll}
\min _{(\mathbf{K}, \mathbf{S})} & \operatorname{Tr}\left(\mathbf{R}^{1 / 2} \mathbf{G S} \mathbf{S G}^{T} \mathbf{R}^{1 / 2}\right)+\operatorname{Tr}(\mathbf{Q S})-\operatorname{Tr}(\mathbf{G S N})-\operatorname{Tr}\left(\mathbf{N}^{T} \mathbf{S G}^{T}\right), \\
\text { subject to } & \mathbf{A S}-\mathbf{B}_{u} \mathbf{G S}+\mathbf{S A}^{T}-\mathbf{S G}^{T} \mathbf{B}_{u}^{T}+\mathbf{B}_{w} \mathbf{B}_{w}^{T} \leq 0, \quad \mathbf{S}=\mathbf{S}^{T}>0,
\end{array}
$$

where $\operatorname{Tr}()$ is the trace of the matrix. This is not an EVP problem since it is not convex because of the terms involving GS. To obtain a convex version of the problem, two new variables are introduced $\mathbf{X}=\mathbf{R}^{1 / 2} \mathbf{G S G}^{T} \mathbf{R}^{1 / 2}$ and $\mathbf{Y}=\mathbf{G S}$. Substituting these variables into (3.10) and using the transformation given by (3.2) and (3.3), the EVP representation of LQR problem is obtained as

$$
\begin{array}{cc}
\min _{(\mathbf{X}, \mathbf{S}, \mathbf{Y})} & \operatorname{Tr}(\mathbf{X})+\operatorname{Tr}(\mathbf{Q S})-\operatorname{Tr}(\mathbf{Y} \mathbf{N})-\operatorname{Tr}\left(\mathbf{N}^{T} \mathbf{Y}^{T}\right), \\
\text { subject to } & \mathbf{A S}-\mathbf{B}_{u} \mathbf{Y}+\mathbf{S} \mathbf{A}^{T}-\mathbf{Y}^{T} \mathbf{B}_{u}^{T}+\mathbf{B}_{w} \mathbf{B}_{w}^{T} \leq 0 \\
& {\left[\begin{array}{cc}
\mathbf{X} & \mathbf{R}^{1 / 2} \mathbf{Y} \\
\mathbf{Y}^{T} \mathbf{R}^{1 / 2} & \mathbf{S}
\end{array}\right]>0 .}
\end{array}
$$


Table 1: Physics and geometric properties of the truss structures.

\begin{tabular}{lc}
\hline Length of structural element $(\mathrm{mm})$ & 30 \\
Diameter of structural element $(\mathrm{mm})$ & 5 \\
Young's Modulus $(\mathrm{GPa})$ & 210 \\
Density $\left(\mathrm{Kg} / \mathrm{m}^{3}\right)$ & 7800 \\
\hline
\end{tabular}

The optimal control gain is then computed from $\mathbf{G}=\mathbf{Y S}^{-1}$, so $\mathbf{K}=\alpha \mathbf{Y S}^{-1}$. For well-posed problem with no additional constraints, the $\mathbf{S}$ that optimizes (3.11) is identical to the optimal state covariance matrix $\mathbf{P}$. In this paper, LQR in LMI version was implemented using the LMI Toolbox of Matlab.

\subsection{Kalman states estimator}

The Kalman estimator was named after Rudolf E. Kalman, though Thorvald Nicolai Thiele and Peter Swerling actually developed a similar algorithm earlier. Stanley F. Schmidt is generally credited with developing the first implementation of a Kalman estimator. It was during a visit of Kalman to the NASA Ames Research Center that he saw the applicability of his ideas to the problem of trajectory estimation for the Apollo program, leading to its incorporation in the Apollo navigation computer. The estimator was developed in papers by Swerling [14], Kalman [15], and Kalman and Bucy [16]. In control theory, the Kalman estimator is most commonly referred to as Kalman filter or, mainly, as linear quadratic estimation (LQE). In this paper, the Kalman estimator gain was obtained using the software Matlab through command "lqe."

\subsection{Dynamic and modal uncertainties representation}

This paper presents a methodology to design a robust control considering dynamic or modal uncertainties in the state space model. The uncertainty ranges in the parameters were quantified through experimental identification considering different excitations. The mathematical model in state space realization was obtained using the numerical method of subspace identification (N4SID). An expressive part of identification methods concerns with computing polynomial models, which, typically, give rise to numerically illconditioned mathematical problems, especially for multi-input multioutput systems [17]. N4SID algorithms are then viewed as optimal alternatives. This approach is advantageous, especially for high-order multivariable systems, where the parameterization is not trivial. The parameterization is needed to start up classical identification algorithms, which means that a priori knowledge of the order and of the observability or controllability indices is required [18].

Using $n_{t}\left(n_{t}>1\right)$ data acquisition tests, it is possible to realize $n_{t}$ model identification (through N4SID algorithm) and, consequently, $n_{t}$ dynamic models. Each one can be used as a polytopic vertex, $S_{i}$ (see (2.5)). In this way, it is possible to define the polype $\Omega$ to describe the convex space $C o$. Considering this convex space to solve the controller, it is possible to obtain a robust gain, and so to get a controller with the ability for maintaining satisfactory stability and performance features in the presence of parameters uncertainties and variations. 


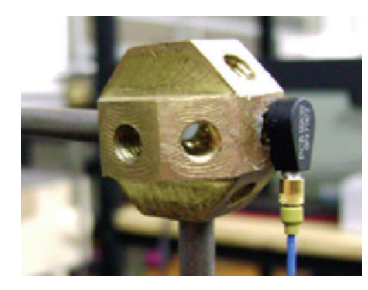

(a)

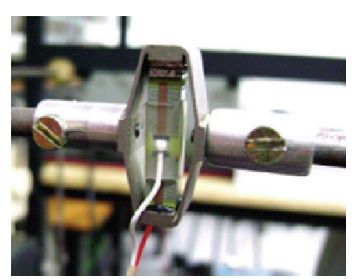

(b)

Figure 2: Accelerometer in joint 4 and details of the PZT wafer stack actuator.

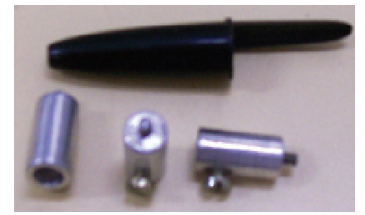

(a)

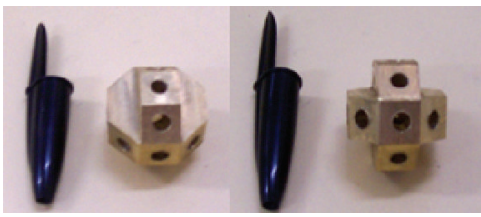

(b)

Figure 3: (a) Adapters to connect PZT stacks; (b) structural nodes.

\section{Experimental control design}

The proposed methodology was experimentally applied in a 3D truss structure, as shown in Figure 1. The physics and geometric properties of the truss are given in Table 1. The properties of the PZT wafer stacks elements are shown in Table 2, and the output signals were obtained with an accelerometer, model 352C22 PCB Piezotronics. Figures 2(a) and 2(b) show the accelerometer in joint 4 , and the detail of the PZT wafer actuator, respectively. In this application, the PZT 1 was used to apply the disturbance input, $\mathbf{w}(t)$, and PZT 2 was used as control force input, $\mathbf{u}(t)$.

The connections of the piezoelectric actuators in the structural elements were made through adapters, shown in Figure 3(a). Figure 2(b) shows details of this connection. These adapters were made using aluminum rod in order to connect the structural part and the PZT wafer actuator. This kind of actuator amplifies the displacement in the axial direction of the structural member, and it is named active member. The joint connections were made of copper, with $24 \mathrm{~mm}$ of diameter in the geometric format of eight sides, as shown in Figure 3(b).

The dynamic model represented through state space realization was identified using the N4SID algorithm considering $n_{t}=6$. Therefore, the dynamic uncertainties were considered through identification of six models, then, the convex space was obtained with six vertexes $\left(S_{1}, S_{2}, \ldots, S_{6}\right)$. The order of the model (dimension of state vector) was chosen as 2 , so the first mode was identified. Using the first identified mode, the Kalman estimator gain was computed by the "lqe" command of the Matlab software. It was computed as $\mathbf{L}=[463,998265,8124]^{T}$. Considering the weighting matrices $\mathbf{Q}$ and $\mathbf{R}$ as $5 * \mathbf{I}$ and $1 * \mathbf{I}$, respectively, where $\mathbf{I}$ is the identity matrix, the controller gain was obtained as $\mathbf{G}=$ $[-1,54262,5514]$. The scalar amplifier $\alpha$ was chosen as 80 to the first mode. The controller was designed to the first mode, however, at the practice test, it was verified that the second mode also had a significant attenuation in the vibration amplitude. 
Table 2: Physics and geometric properties of PZT wafer stack actuators, based on material designation APA60 M (Amplified Piezo Actuators, CEDRAT).

\begin{tabular}{lcc}
\hline Property & Unit & APA60 M \\
\hline Displacement & $(\mu \mathrm{m})$ & 80 \\
Blocked Force & $(\mathrm{N})$ & 110 \\
Stiffness & $(\mathrm{N} / \mu \mathrm{m})$ & 1,38 \\
Resonance Frequency (Free-Free) & $(\mathrm{Hz})$ & 10400 \\
Response Time (Free-Free) & $(\mathrm{ms})$ & 0,05 \\
Resonance Frequency (Blocked-Free) & $(\mathrm{Hz})$ & 2800 \\
Response Time (Blocked-Free) & $(\mathrm{ms})$ & 0,18 \\
Voltage Range & $(\mathrm{V})$ & $-20 \cdots 150$ \\
Capacitance & $(\mu \mathrm{F})$ & 1,55 \\
Resolution & $(\mathrm{nm})$ & 0,8 \\
Thermo-Mechanical Behaviour & $(\mu \mathrm{m} / \mathrm{K})$ & 1,02 \\
Height H (in actuation direction) & $(\mathrm{mm})$ & 13,0 \\
Length & $(\mathrm{mm})$ & 26,9 \\
Width (ind. Edges, wires) & $(\mathrm{mm})$ & 11,5 \\
Mass & $(\mathrm{g})$ & 20,0 \\
\hline Standard Mechanical Interface $[\mathrm{TH}]$ & 2 flat surface $5 * 10 \mathrm{~mm}^{2}$ with M2.5 threaded hole \\
Standard Electrical Interface & 2 PTFE insulated AWG30 wires 100 mm long with ø 1 banana plug \\
\hline
\end{tabular}

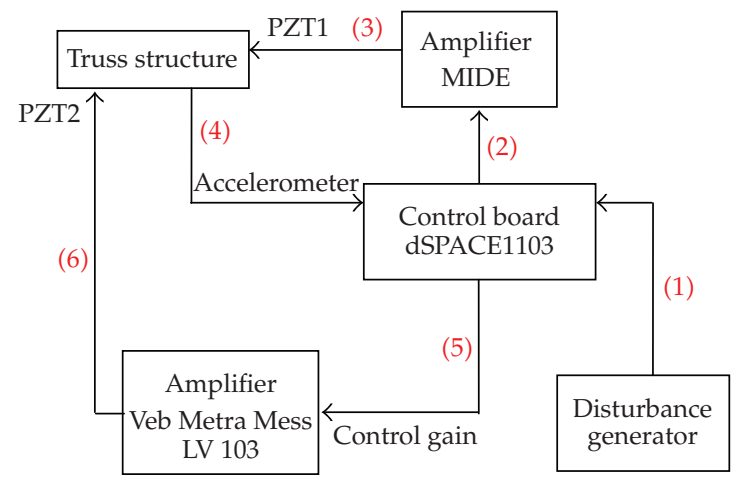

Figure 4: Disposition of the experimental setup.

To verify the results of the active vibration control, two cases were considered. Figure 4 shows the configuration of the experimental setup used. In the first case, the disturbance input was a sine signal with frequency of $16 \mathrm{~Hz}$ (approximately the first natural frequency). Figure 5 shows the output signal with and without control obtained in joint 4 . Figure 6 shows the experimental output measured using the accelerometer in joint 4 and the estimated output through Kalman estimator algorithm. These results were obtained using the dSPACE 1103 control board and the Simulink/Matlab.

In the second case, a disturbance input was considered as a sine signal with frequency of $26 \mathrm{~Hz}$ (approximately the second natural frequency). Figure 7 shows the response in time domain for the uncontrolled and controlled systems. The controller was applied approximately after 4.5 seconds. Figures 8 and 9 show the control force in the PZT stack 


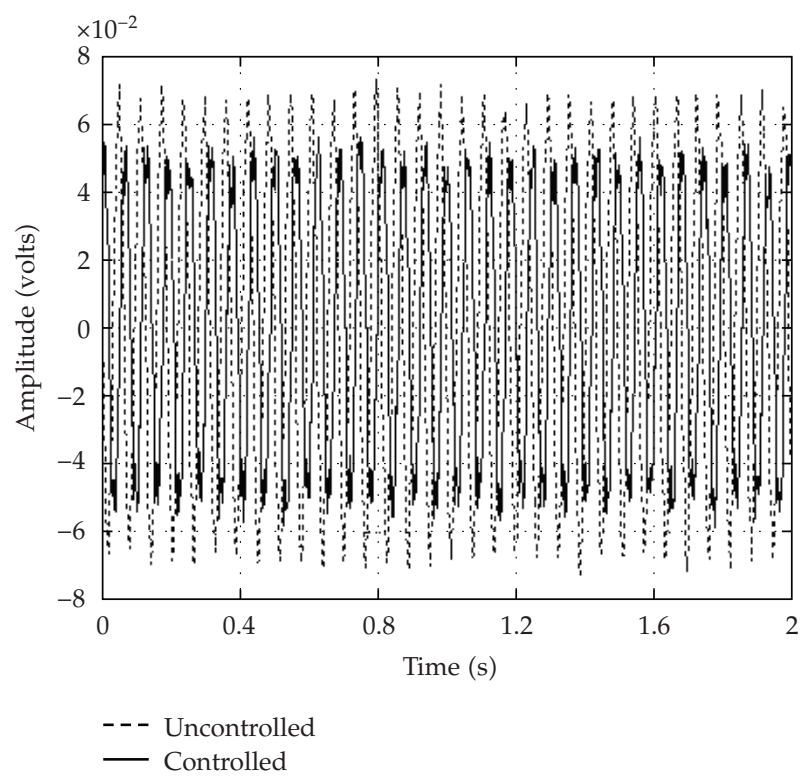

Figure 5: Output signal measured in the joint 4 using accelerometer-controlled and uncontrolled systems.

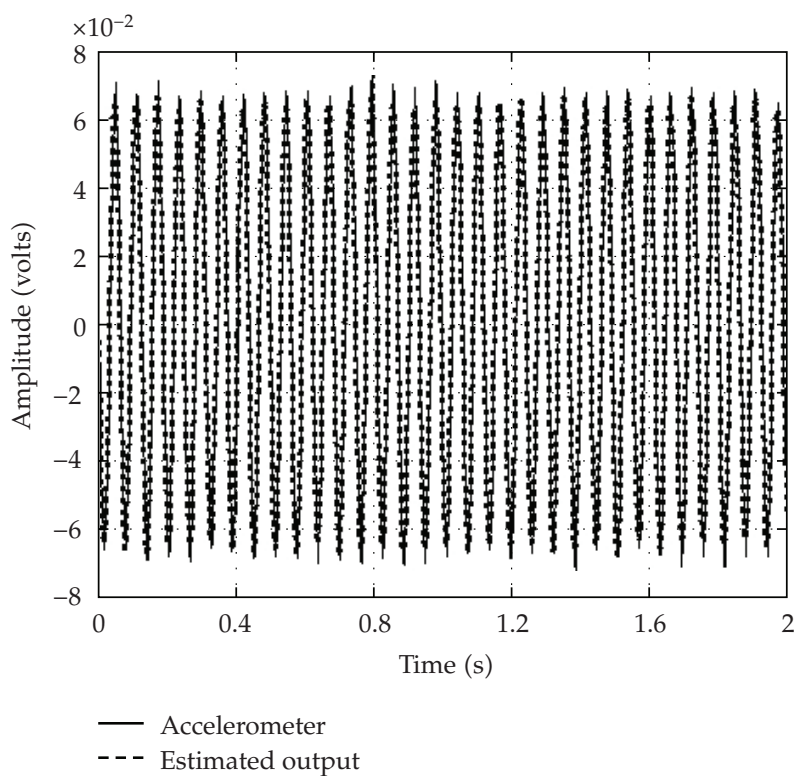

Figure 6: Estimated output signal in joint 4 through Kalman Estimator and experimental output.

actuator 2 and the output estimated through Kalman estimator, respectively. Figure 10 shows the frequency response function (FRF) of the uncontrolled and controlled truss structures. It was attenuated approximately by $6 \mathrm{~dB}$ and $9 \mathrm{~dB}$ to the first and second modes, respectively. 


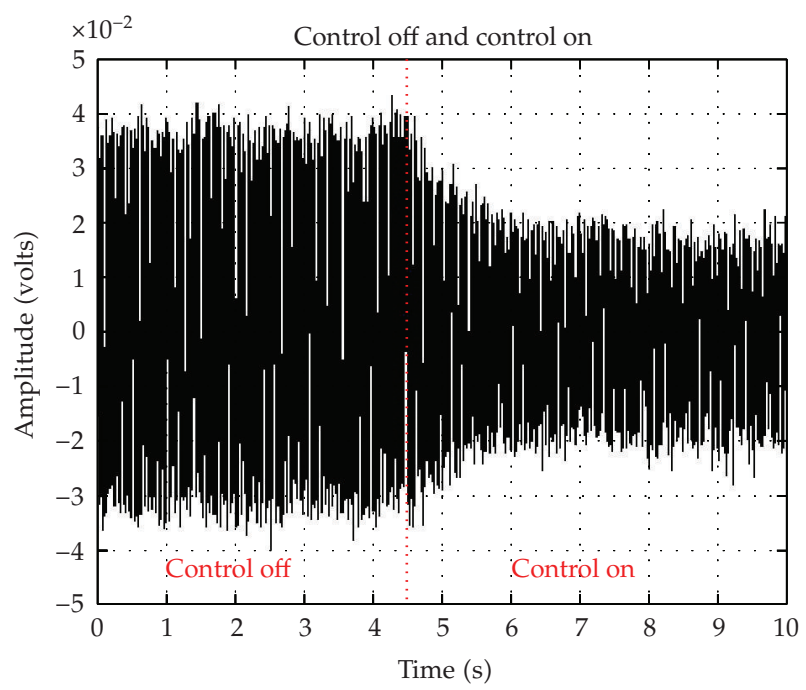

Figure 7: Experimental output signal of uncontrolled and controlled truss structure in joint 4.

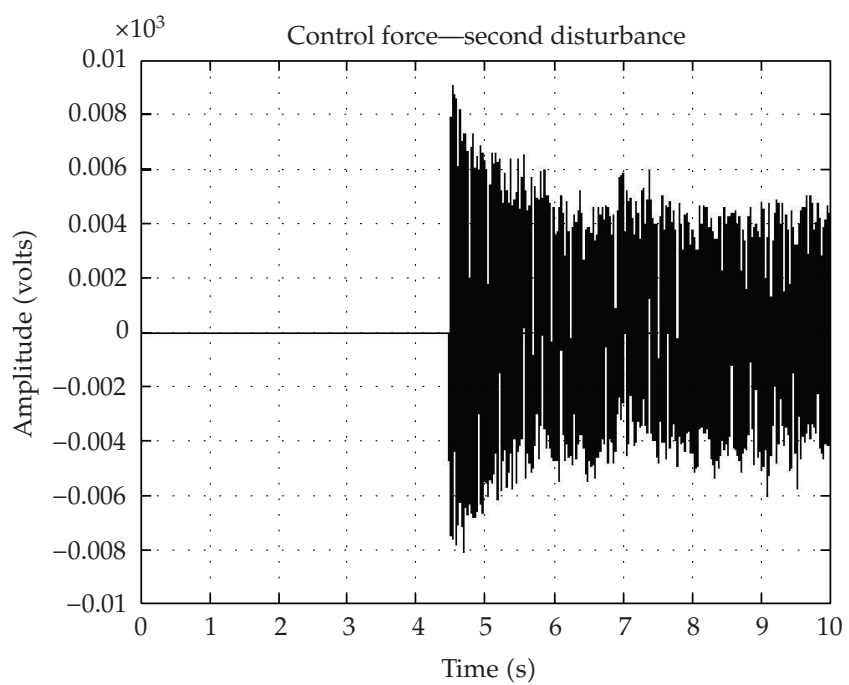

Figure 8: Control force applied by PZT wafer stack 2 for the second case of disturbance.

\section{Final remarks}

Over the last two decades, the use of piezoceramics as actuators and sensors has increased considerably, since they provide an effective means of high-quality actuation and sensing mechanism. Piezoceramics have been considered as an alternative due features as low-cost, light weight, and easy-to-implement for active control of structural vibration.

In this paper, the subspace identification method was used to obtain the parameters of the system and to characterize the uncertainty ranges present in the model. In the experimental application, the uncertainties were defined in a polytopic with six vertexes. The system identification technique was used to identify the model in the state space 


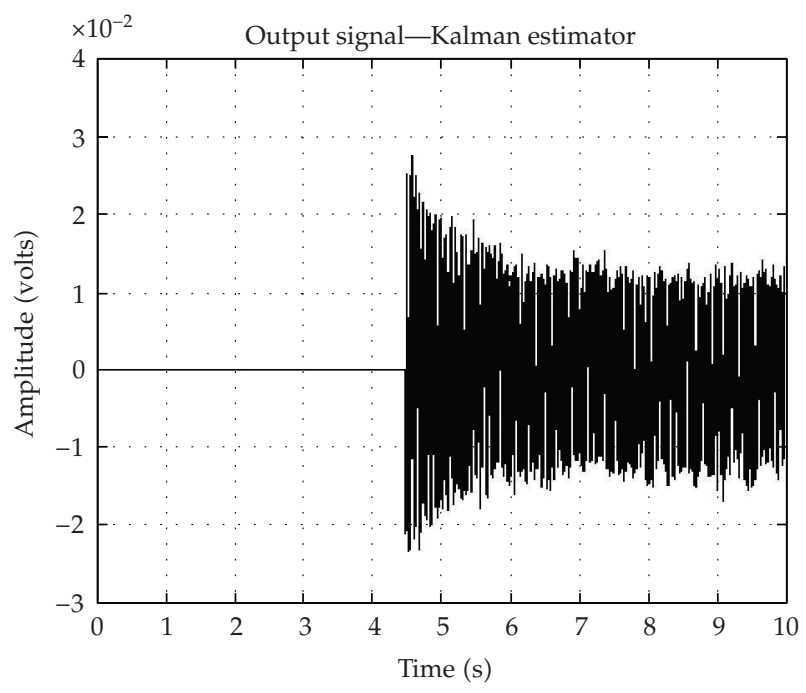

Figure 9: Output signal estimated through Kalman Estimator algorithm for the second case of disturbance (sine signal with $26 \mathrm{~Hz}$ ).

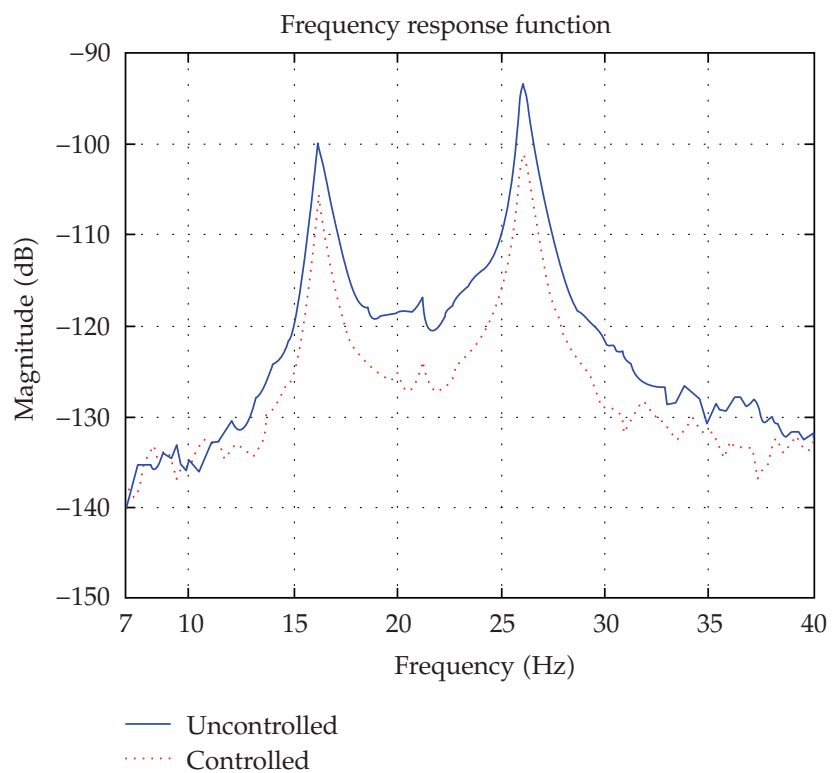

Figure 10: Frequency response function of uncontrolled and controlled system.

realization that was converted to modal coordinates. The LQR controller solved through LMI was experimentally implemented and applied in a 3D truss structure that contains nonlinearities and uncertainties. The disturbance was applied through PZT wafer stack. LMI techniques that are classified by some authors as postmodern control present many advantages, mainly due to the facilities of solving numerical problems for complex structure, where the analytical solution should be difficult to implement. Uncertainties in the dynamic matrix were considered in order to design a robust active vibration control. However, any 
other uncertain parameter should be added, for instance, damping coefficients. In this case, it is only needed to consider new vertexes in the box with all uncertain parameters and write the respective LMIs. The proposed approach showed that an efficient robust controller design can be obtained for complex structures with nonlinearities and uncertainties.

\section{Acknowledgment}

The authors acknowledge the support of the Research Foundation of the State of São Paulo (FAPESP, Brazil).

\section{References}

[1] E. J. Ruggiero, Active dynamic analysis and vibration control of gossamer structures using smart materials, M.S. thesis, Virginia Polytechnic Institute and State University, Blacksburg, Va, USA, 2002.

[2] Y. J. Yan and L. H. Yam, "A synthetic analysis on design of optimum control for an optimized intelligent structure," Journal of Sound and Vibration, vol. 249, no. 4, pp. 775-784, 2002.

[3] K. M. Grigoriadis and R. E. Skelton, "Low-order control design for LMI problems using alternating projection methods," Automatica, vol. 32, no. 8, pp. 1117-1125, 1996.

[4] S. Boyd, L. El Ghaoui, E. Feron, and V. Balakrishnan, Linear Matrix Inequalities in System and Control Theory, vol. 15 of SIAM Studies in Applied Mathematics, SIAM, Philadelphia, Pa, USA, 1994.

[5] P. Gahinet, A. Nemirovski, A. J. Laub, and M. Chiliali, "LMI Control Toolbox User's Guide," The Mathworks Inc., Natick, Mass, USA, 1995.

[6] F. Sarracini Jr. and A. L. Serpa, "Reduced model in $\mathrm{H}^{\infty}$ vibration control using linear matrix inequalities," Shock and Vibration, vol. 13, no. 4-5, pp. 469-484, 2006.

[7] S. Da Silva, V. Lopes Jr., and M. J. Brennan, "Design of a control system using linear matrix inequalities for the active vibration control of a plate," Journal of Intelligent Material Systems and Structures, vol. 17, no. 1, pp. 81-93, 2006.

[8] Y. Chen and C. Zhu, "Active vibration control based on linear matrix inequality for rotor system under seismic excitation," Journal of Sound and Vibration, vol. 314, no. 1-2, pp. 53-69, 2008.

[9] W. K. Gawronski, Dynamics and Control of Structures: A Modal Approach, Mechanical Engineering Series, Springer, New York, NY, USA, 1998.

[10] R. E. Kalman, "Contributions to the theory of optimal control," Boletín de la Sociedad Matemática Mexicana, vol. 5, pp. 102-119, 1960.

[11] A. Kumar, F. Wu, M. Lin, et al., "Potential Applications of Smart Layer ${ }^{\circledR}$ Technology for Homeland Security," Acellent Technologies, Inc. 155 C-3 Moffett Park Drive, Sunnyvale, CA e National Institute of Standards and Technology, 2004.

[12] K. Ogata, Modern Control Engineering, Pretince Hall, Upper Saddle River, NJ, USA, 1997.

[13] E. A. Johnson and B. Erkus, "Structural control with dissipative damping devices," in Proceedings of the American Control Conference (ACC '02), vol. 3, pp. 2463-2468, Anchorage, Alaska, USA, May 2002.

[14] P. Swerling, "A proposed stagewise differential correction procedure for satellite tracking and prediction," Tech. Rep. P-1292, RAND, Santa Monica, Calif, USA, January 1958.

[15] R. E. Kalman, "A new approach to linear filtering and prediction problems," Journal of Basic Engineering, vol. 82, pp. 35-45, 1960.

[16] R. E. Kalman and R. S. Bucy, "New results in linear filtering and prediction theory," Journal of Basic Engineering, vol. 83, pp. 95-108, 1961.

[17] P. Van Overschee and B. De Moor, "N4SID: subspace algorithms for the identification of combined deterministic-stochastic systems," Automatica, vol. 30, no. 1, pp. 75-93, 1994.

[18] L. Ljung, System Identification: Theory for the User, Prentice Hall Information and System Sciences Series, Pretince Hall, Upper Saddle River, NJ, USA, 1987. 


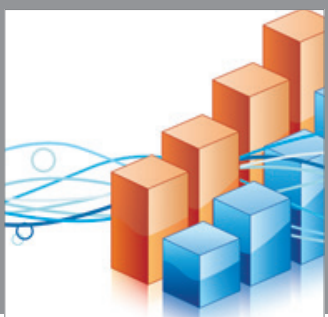

Advances in

Operations Research

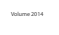

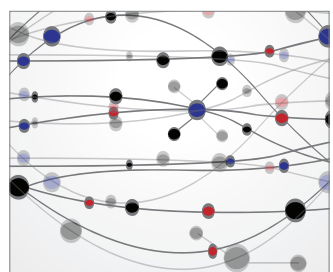

\section{The Scientific} World Journal
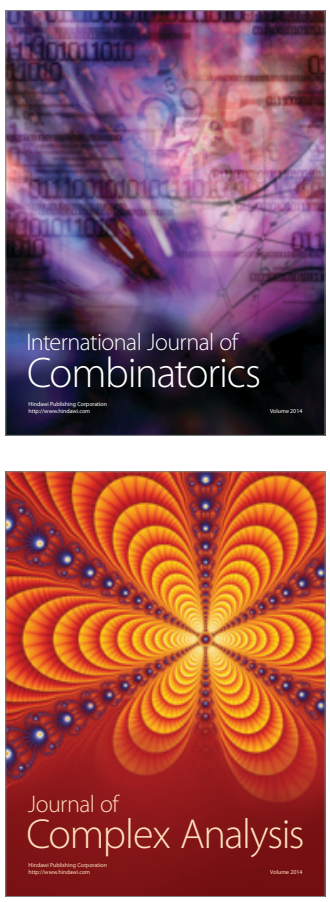

International Journal of

Mathematics and

Mathematical

Sciences
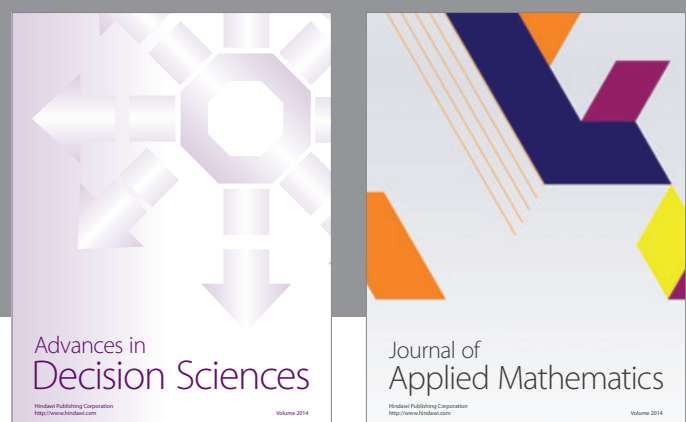

Journal of

Applied Mathematics
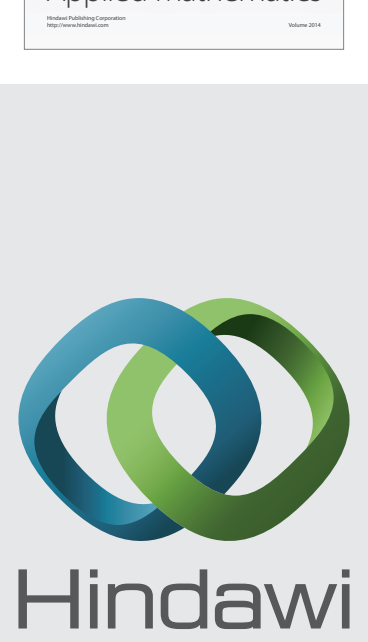

Submit your manuscripts at http://www.hindawi.com
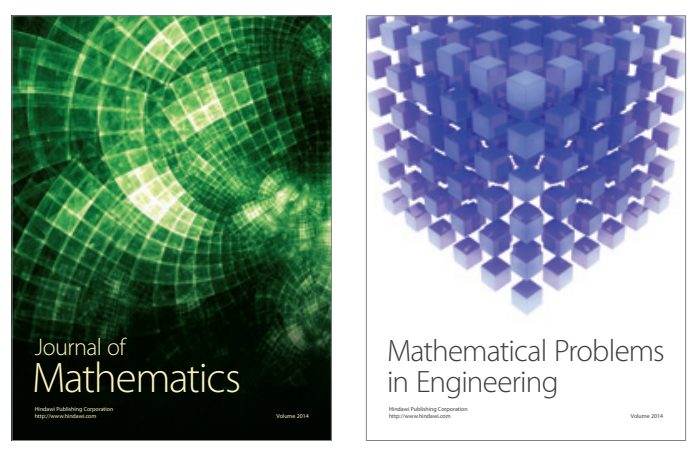

Mathematical Problems in Engineering
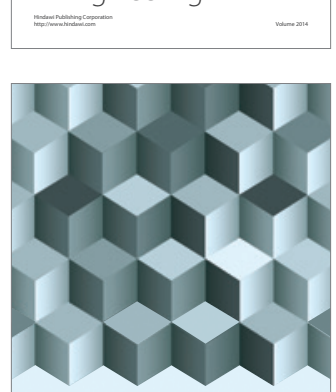

Journal of

Function Spaces
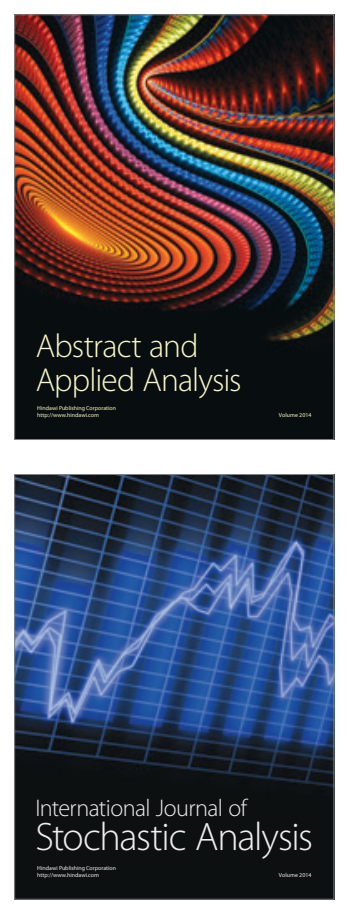

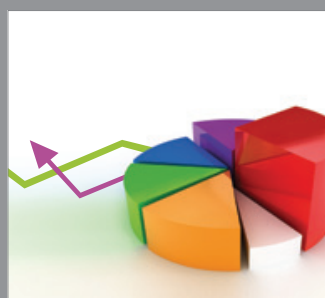

ournal of

Probability and Statistics

Promensencen
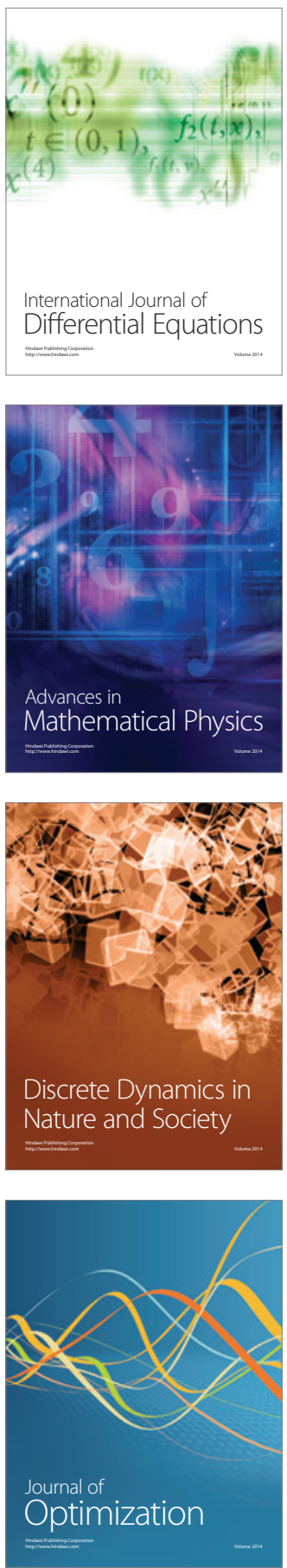